\title{
Gravitational self-localization in quantum measurement
}

\author{
Tamás Geszti \\ Department of the Physics of Complex Systems, \\ Eötvös University; H-1117 Budapest, Hungary \\ e-mail: geszti@galahad.elte.hu
}

\begin{abstract}
Within Newton-Schrödinger quantum mechanics which allows gravitational self-interaction, it is shown that a no-split no-collapse measurement scenario is possible. A macroscopic pointer moves at low acceleration, controlled by the Ehrenfest-averaged force acting on it. That makes classicality self-sustaining, resolves Everett's paradox, and outlines a way to spontaneous emergence of quantum randomness. Numerical estimates indicate that enhanced short-range gravitational forces are needed for the scenario to work. The scheme fails to explain quantum nonlocality, including two-detector anticorrelations, which points towards the need of a nonlocal modification of the Newton-Schrödinger coupling scheme.
\end{abstract}

PACS numbers: 03.65.-w, 03.65.Ta, 04.40.-b

\section{INTRODUCTION}

If microscopic quantum mechanics and macroscopic classical mechanics are limiting cases of the same dynamic law, that law cannot be the linear, deterministic Schrödinger equation: the quantum measurement process, with its random switch governed by squared amplitudes is manifestly nonlinear, and apparently nondeterministic [1]. However, in all the rest of quantum phenomena, nonlinearity is extremely elusive. The concept of collapse (reduction) of the wave function [2], the applicability of which is limited to the act of quantum measurement, is a proper expression of that situation.

As an attempt to find a unified dynamical law interpolating between quantum and classical realms, modified quantum mechanics schemes of the so-called stochastic reduction type have been developed 3], which add both a nonlinearity and a random force to standard quantum mechanics; those schemes are sometimes related to gravity 4]. Along with that line of thinking, there are continuing attempts to understand the effects of nonlinearity alone [5], without built-in randomness. Besides calculating eventual detectable modifications of spectra, such studies are usually focussed on demonstrating the stabilization of soliton-like motions against the spreading of wave packets predicted by linear quantum mechanics: that stabilization is tentatively identified with emerging classicality. Nonlinearities which are too weak to show up mechanically, might prove strong enough to be detected by interferometry [6].

In what follows, we remain with the special case of an attractive nonlinearity provided by gravity, acting in a mean-field way [7]: dynamics is governed by Schrödinger's equation, in which gravity appears as an external, classical field $\Phi$, obeying the Poisson equation

$$
\nabla^{2} \Phi(\vec{r})=4 \pi G \varrho(\vec{r})
$$

where $G$ is Newton's constant of gravity. The source term is the quantum mechanical mean value of the operator of mass density in quantum state $|\Psi\rangle$ for particles of masses $m_{i}$ and position operators $\hat{\vec{r}}_{i}$ :

$$
\varrho(\vec{r})=\left\langle\Psi\left|\sum_{i} m_{i} \delta\left(\vec{r}-\hat{\vec{r}}_{i}\right)\right| \Psi\right\rangle .
$$

The $|\Psi\rangle$-dependence of the field $\Phi$ makes the Schrödinger equation nonlinear. In recent times, such a scheme is called Newton-Schrödinger quantum mechanics, and considered a promising starting point to attack the problem of quantum state reduction.

In the present paper we pursue that line of reasoning, and focus our attention to the situation of quantum measurement on a superposition, which is a special situation in that the apparatus is actively driven towards different positions. That poses much more stringent requirements on the attractive nonlinearity than the stabilization of a single wave packet does. Gravity-based nonlinearity [7] is far more efficient in such a situation than any local one [5], because of the long-range attraction between split components of a wave packet. A further crucial point is that the strength of gravity is tested against acceleration; in quantum measurement microscopic bodies act on macroscopic ones, and the acceleration of the latter is low.

Diósi 7] poses the question whether self-gravity excludes persistently split wave packets, and answers no. Here we pose a different question: can self-gravity exclude the creation of split wave packets under some circumstances; in particular, can it successfully protect macroscopic bodies against macroscopic splitting, even in quantum measurement situations? As shown below, now the answer can be yes, if the bodies are massive enough and the accelerations are low. The detailed condition is derived below, see Eq. (6).

If that condition is satisfied by macroscopic bodies, the consequences are far-reaching and beneficial for the theory. Classicality, once generated [8], may become selfsustaining. The nature of the quantum-classical border the Bohrean cut - is immediately outlined: gravitational self-interaction is weak on microscopic objects, strong on macroscopic ones, and the two can be distinguished by means of a physical criterion. Having a single dynam- 
ical law, Everett's no-collapse scheme [9] is recovered, without its paradoxical features: for a macroscopic body (e.g. a detector) the split components of the wave function remain glued to each other, in accordance with everyday experience. Viewed from the macroscopic side, this is effectively a no-split no-collapse scheme, in which Schrödinger's cat remains doubly-dead or doubly-live. The Schrödinger wave field gets "materialized", being the source of gravity, thereby eliminating the necessity of philosophical discussions about epistemological vs. ontological status of the state vector. In addition, the scheme is suggesting dynamical mechanisms about generation of randomness in the emerging classical domain, in accordance with Born's rule of probabilities [10].

In spite of that attractive view opening up, there are important reasons to doubt in the validity of the NewtonSchrödinger scheme. First is the weakness of gravity as we know it from macroscopic measurements: the numerical estimates presented below require a one-millimeter size of atomic condensed matter, and astronomical sizes of electron liquid to behave classically in a quantum measurement situation. If gravity proved to be strongly enhanced with respect to Newton's law for short distances, the chances of the scheme would be much better; theoretical indications that such short-range enhancement may be real are briefly summarized in the text below.

There is a more serious limitation of principle too: all of the analysis in the next Section refers to the case of a single detector. As soon as several detectors are probing an entangled system [11] or only but a single microobject [12], the scheme fails to describe the much-tested quantum correlations manifest in multi-detector anticoincidences, losing even the formal explanation offered by linear quantum mechanics.

Below we will conclude that the tender spot of the scheme, responsible for those important failures, is the way we characterize the source of gravity. The obvious choice of Eq. (2), used in all the work listed under Ref. 7], may turn out to be wrong. Some hints about the desired modification will be discussed at the end of this paper.

The main text below begins by deriving an effective gravitational self-interaction potential that appears in the c.o.m. Schrödinger equation of a macroscopic body. The derivation concludes in formulating the classicality condition, Eq. (6). Then we continue by analyzing a minimum model of the measurement process, and demonstrate the way module-squared amplitudes control the macroscopic dynamics, offering a dynamical route to Born's rule. Then numerical estimates are presented, indicating that standard gravity is just strong enough to make the above picture valid for macroscopic condensed matter, however, it is far too weak for all-electronic particle detection systems. The analysis of entangled detectors and an outlook section conclude the paper.

\section{THE GRAVITATIONAL SELF-INTERACTION OF MACROSCOPIC BODIES}

Following Diósi 7], the detector is depicted as a rigid body of mass density $\varrho_{0}$ and global mass $M$, the quantum state of which can be separated into a frozen internal state and a c.o.m. wave function $\psi(\vec{r}, t)$. The latter obeys a c.o.m. Schrödinger equation containing the potential energy $V_{g r}(\vec{r})$ of the body, generated by itself at some place $\vec{r}^{\prime}$, averaged over $\left|\psi\left(\vec{r}^{\prime}, t\right)\right|^{2}$ according to Eq. (2). Using Diósi's result for the non-averaged interaction of two almost overlapping spheres of radius $R$, as long as spreading and splitting of the c.o.m. wave packet are small: $\left|\vec{r}-\vec{r}^{\prime}\right| \ll R$, we obtain the simple quadratic expression

$$
\begin{array}{r}
V_{g r}(\vec{r}) \approx \int d \vec{r}^{\prime}\left(V_{g r}^{0}+\frac{1}{2} M \omega_{g r}^{2}\left|\vec{r}-\vec{r}^{\prime}\right|^{2}\right)\left|\psi\left(\vec{r}^{\prime}, t\right)\right|^{2} \\
=V_{g r}^{0}+\frac{1}{2} M \omega_{g r}^{2}\left|\vec{r}-\vec{r}^{*}\right|^{2}+\frac{1}{2} M \omega_{g r}^{2} \Delta^{2}
\end{array}
$$

where $\vec{r}^{*}$ and $\Delta(\ll R)$ are respectively the mean position and the standard deviation of $|\psi(\vec{r})|^{2}$,

$$
V_{g r}^{0}=-\frac{6}{5} \frac{G M^{2}}{R}
$$

and

$$
\omega_{g r}^{2}=G \varrho_{0}
$$

The analogous expressions for axial displacements in a slab-like geometry are presented in the Appendix.

The last term in Eq. (3) is a time-dependent energy shift, giving rise to a global phase factor, observable in principle by interferometry as a scalar Aharonov-Bohm effect [13].

The c.o.m. wave packet $\psi(\vec{r}, t)$, split or not, is expected to carry out all kinds of oscillatory motion in the harmonic potential (3). As a rough estimate, $\omega_{g r}^{2} R$ is the maximum confining acceleration provided by gravitational self-attraction: that gives the classicality criterion

$$
\omega_{g r}^{2} R \gg a_{\max }
$$

where $a_{\max }$ is the maximum deconfining acceleration the apparatus undergoes during the measurement process. In view of Eq. (5), this can be regarded as a criterion for the minimum size of the apparatus to behave classically. Having a non-relativistic description at hand, the Planck scale is irrelevant in this respect; however, as seen below, the density of condensed matter points to a well-defined size range.

Eq. (6) is subjected to numerical estimates below. However, before that, we assume that the criterion is met and analyze its consequences concerning quantum measurement. 


\section{MODEL OF THE MEASUREMENT PROCESS}

\section{A. Emergence of the averaged force}

As a minimum model of the compound system object+apparatus, we investigate the measurement of the quasi-spin observable $\hat{\sigma}_{z}$ on a two-state microobject with orthogonal basis states $|+\rangle$ and $|-\rangle$. Throughout the measurement, the state $|\Psi\rangle$ of the compound system is represented by the quasi-linear expression

$$
\begin{aligned}
& \langle\vec{r} \mid \Psi\rangle=\left(c_{+}|+\rangle+c_{-}|-\rangle\right) \psi(\vec{r}, 0) \\
& \quad \Rightarrow c_{+}|+\rangle \psi_{+}(\vec{r}, t)+c_{-}|-\rangle \psi_{-}(\vec{r}, t) .
\end{aligned}
$$

Nonlinearity enters through the dynamics of the split partial waves $\psi_{ \pm}(\vec{r}, t)$.

Measurement is brought about by a spin-dependent interaction

$$
\hat{H}_{\text {meas }}=\hat{H}_{0}+|+\rangle\left\langle+\left|V_{\text {meas }}^{+}(\vec{r})+\right|-\right\rangle\langle-| V_{\text {meas }}^{-}(\vec{r}),
$$

where the measurement interactions $V_{\text {meas }}^{ \pm}$induce spindependent dynamics of the c.o.m. wave function, tending to drive the two components $\psi_{ \pm}(\vec{r}, t)$ of the detector apart from each other, whereas the spin-independent operator

$$
\hat{H}_{0}=-\frac{\hbar^{2}}{2 M} \nabla^{2}+V_{\text {meas }}^{0}(\vec{r})+V_{g r}(\vec{r})
$$

contains - besides the inactive apparatus terms - the gravitational self-interaction of the detector, counteracting both the spreading of each partial wave component $\psi_{ \pm}(\vec{r}, t)$ and their separation under the action of $V_{\text {meas }}^{ \pm}$. As long as both effects remain in the range $r \ll R$, Eq.(3) remains valid, $\vec{r}^{*}$ being now the common c.o.m of the split wave packets.

In view of the entanglement of the two partial wave packets of the apparatus to two orthogonal states of the microobject 14], $\psi_{+}$and $\psi_{-}$can be considered as two classical objects centered around $\vec{r}+$ and $\vec{r}-$ resp., and their dynamics can be directly inferred from Ehrenfest's theorem. According to that theorem, accelerations are controlled by the quantum mechanical mean values of the forces corresponding to various terms in Eq. (8). The averages should be calculated in state (7). In particular, the gravitational forces that glue the partial wave packets together cancel out for the compound object, in accordance with Newton's 3rd law:

$\mathcal{F}_{g r}=-M \omega_{g r}^{2}\left(\left|c_{+}\right|^{2}\left(\vec{r}^{+}-\vec{r}^{*}\right)+\left|c_{-}\right|^{2}\left(\vec{r}^{-}-\vec{r}^{*}\right)\right)=0$

in view of the definition of the c.o.m. Therefore the acceleration of the bound double wave packet as a whole is controlled by the quantum mechanical average of the non-gravitational forces:

$$
\mathcal{F}_{\text {meas }}=-\nabla\left(V_{\text {meas }}^{0}+\left|c_{+}\right|^{2} V_{\text {meas }}^{+}+\left|c_{-}\right|^{2} V_{\text {meas }}^{-}\right) .
$$

This important result says that in the classical domain the dynamics of the measuring apparatus is governed by modulus-squared quantum amplitudes acting as external classical fields, for unlimited time scales.

\section{B. Generation of randomness}

Since classical mechanical systems are capable to generate random behavior through a number of mechanisms, Eq. (11) is certainly a valueable clue to the understanding of Born's law of probabilities. As a matter of fact, systems responding to external fields in a linear way are plentiful around us: any device of sufficiently strong ohmic-stochastic response is a quantum detector obeying Born's rule.

Our schematic models cannot be applied to real detectors without considering their two-stage, photographic mode of operation: fast passage of a particle acts as a kind of exposure, leaving a latent trace in the form of ionization or excitation, fully within the range of linear quantum mechanics; that trace is subsequently developed into a macroscopic signal at a comfortable pace, open to gravitational control. Either stage can exhibit some mechanism of amplification, often based on metastability [15]. In a photomultiplier or avalanche photodiode [16] the first stage is the avalanche formation itself, the second the rise of a voltage pulse in the external circuit; in a cloud (or bubble) chamber the first is the formation of a trace of ionization [17], the second the condensation of liquid droplets (or bubble formation in a superheated liquid). The exposure and development stages are obviously associated to different degrees of freedom.

In what follows, we leave those complications and turn back to a highly schematic model for a metastable detector, expected to give a signal for, say, $\sigma_{z}=+1$, triggered by the Ehrenfest-averaged measurement force $\mathcal{F}=-\left|c_{+}\right|^{2} \nabla V_{\text {meas }}^{+}$. As a specific feature of our model, capable to exhibit eventual deviations from Born's rule, we assume that our detector is biased to threshold.

Accordingly, we consider a potential well open along some axis $\xi$ ("measurement coordinate") through a saddle point of the potential:

$$
V_{\text {meas }}^{0}(\vec{r})=a \xi-b \xi^{3}+c\left(r_{\perp}^{2}\right)
$$

where $r_{\perp}$ is the orthogonal distance from axis $\xi$. Classically, for a given energy of the detector, this is a chaotic billiard; closed if its energy is below the height of the saddle, open if not. In the threshold case, in absence of interaction with the microobject the energy should be at the saddle. As interaction starts, the mean measurement force $\mathcal{F}$ would lower the saddle point to first order by $(a / 3 b)^{1 / 2} \mathcal{F}$, and admit barrier crossing inside the circle

$$
r_{\perp} \leq\left(\frac{a}{3 b c^{2}}\right)^{1 / 4} \mathcal{F}^{1 / 2}
$$


at a speed bounded by

$$
v_{\max }=\left(\frac{4 a}{3 b m^{2}}\right)^{1 / 4} \mathcal{F}^{1 / 2} .
$$

Variations of density and speed over the area of the circle cancel out. Finally, the rate of escape from the potential well is obtained as

$$
\Gamma \propto\left(\pi r_{\perp}^{2}\right) \cdot v_{\|}
$$

The first factor being proportional to $\mathcal{F}$, the second to $\mathcal{F}^{1 / 2}$, the escape rate and thereby the probability of detection becomes proportional to the $3 / 2$-th power of $\left|c_{+}\right|^{2}$, which - although qualitatively in the usual way does not agree with Born's rule.

By good luck, real detectors do not operate in that way: they are biased high above threshold, and their signals are cut by some electronic discriminator. That linearizes the $\left|c_{+}\right|^{2}$ dependence and Born's rule is recovered.

Nevertheless, there is a lesson to be drawn from the above derivation, notwithstanding the oversimplified character of the model: Born's rule of strict proportionality between detection probabilities and modulus-squared amplitudes may depend on details of the underlying nonlinear dynamics. Particle detection at very low quantum amplitudes (produced by very asymmetric beamsplitters e.g. along the line of Ref. (18), accompanied by an exploration of non-optimized bias and discrimination levels, possibly extended to EPR-like tests [11] to see if remote detector correlations surpass Born's rule in robustness, is a promising target to detect eventual deviations.

\section{NUMERICAL ESTIMATES}

Now we turn back to the question of whether gravity is strong enough to enforce classicality as outlined in the Introduction. According to Eq. (6), that depends on the accelerations gravitational confinement can hold out.

As a first test, we choose a spherical body of mass density $\varrho_{0} \simeq 10^{4} \mathrm{Kg} / \mathrm{m}^{3}$, typical for condensed matter, down to molecules. Then, an acceleration of $1 \mathrm{~nm} /(1 \mathrm{~s})^{2}$, easy to detect e.g. in an Atomic Force Microscope, gives a size limit $R \gg 1 \mathrm{~mm}$ and a corresponding mass $M \gg$ $10^{-2} g$ as a condition for gravity-stabilized classicality, which - although somewhat too large - seems to be on the borderline of reasonability.

That was the best case. The most widespread case of a real quantum measurement process, all-electronic detection of particles, is the worse. The most massive accelerating component is the electron liquid of electron density $10^{30} / \mathrm{m}^{3}$ and corresponding mass density $\simeq 1 \mathrm{Kg} / \mathrm{m}^{3}$ in the detector - to - voltmeter leads, and it is far from satisfying criterion (6). As a matter of fact, in Geiger mode detection, that electron liquid transfers the charge of an avalanche of $10^{7}$ electrons to the voltmeter through a lead of cross-section $10^{-9} \mathrm{~m}^{2}$ within $10^{-8} \mathrm{~s}$, which cor- responds to $a_{\max }=10^{2} \mathrm{~m} / \mathrm{s}^{2}$, resulting in astronomical sizes required. The geometrical factors referring to non-spherical shapes, presented in the Appendix, offer no sizeable modification.

One important point can save the scheme from being rejected on the basis of the above numerical estimates: some recent theoretical developments indicate that in the submillimeter range new gravitation-like forces may arise, stronger than Newtonian gravity by factors up to $10^{6}$. The effect would be due to large extra dimensions [19], with an eventual further contribution from low-energy supersymmetry breaking [20]. Experiments have found no evidence so far [21] but continue to explore shorter distances [22].

One last point merits discussion here. As mentioned in the Introduction, the very existence of the non-linear selfinteraction, measured e.g. by the constant term $V_{g r a v}^{0}$ appearing in Eq. (3), is in principle open to interferometric detection through the phase difference between two geometries differing in the respective positions of an absorber [6]. For a given density $\varrho_{0}$ the effect scales with $M^{5 / 3}$; using the above figures and a typical time-of-flight of $10^{-2} s$, as a necessary condition for a phase shift of order unity one obtains $M \approx 10^{-14} \mathrm{Kg}$, which is by ten orders of magnitude heavier than present-day limits of molecule interferometry [23]. A more detailed analysis of interferometric possibilities 24] does not change that conclusion. However, if - as mentioned above - gravity proves much stronger on the nanoscale, that may reopen the issue of interferometric detection.

\section{THE NONLOCALITY ISSUE}

Now we turn to the questions of entanglement-based correlations. To begin with the easy part of it, according to a subtle generic objection to nonlinear versions of quantum mechanics [5], they are liable to give rise to superluminal signaling, either between remote entangled subsystems or between Everettean branches of a superposition [25], thereby breaking the fragile "peaceful coexistence" between quantum mechanics and special relativity 27]. The present scheme is free from that kind of objection: on the one hand, as discussed by Polchinski 25], EPR signaling between entangled partners requires collapse of the wave function which is absent here; on the other hand, Everettean branches for macro-objects are now not separated macroscopically, therefore signaling between them remains harmless [26].

There is, however, a major difficulty of the NewtonSchrödinger scheme: its inability to explain the dynamical origin for the robust manifestations of entanglement through anticorrelations between two or more detectors probing a single quantum system [11, 12].

Orthodox Copenhagen quantum mechanics is free from that difficulty, at the expense of disclaiming to look for a dynamical origin of statistical laws. We illustrate that on the simplest case of 1-particle 2-detector anticorrela- 


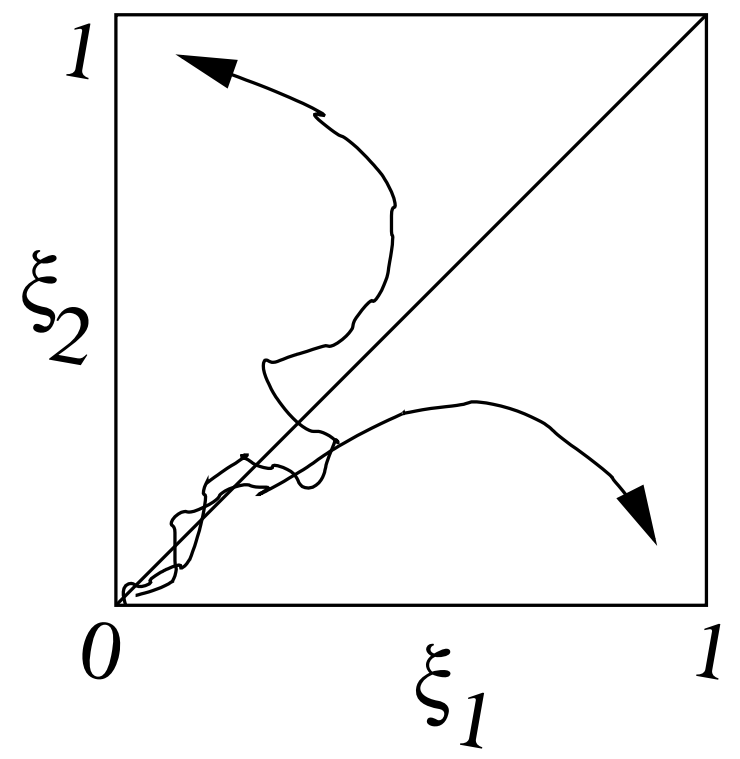

FIG. 1: Anticorrelation of two detectors awaiting one particle: on the two-dimensional flow diagram of the respective measurement coordinates $\xi_{1}$ and $\xi_{2}$ of the detectors (0 if quiescent, 1 if active), there seems to be some force, destabilizing the diagonal and driving the system towards one of the possible end points with one detector active, one quiescent.

tions, i.e. the fact that out of two detectors awaiting a single particle, only one would give a signal [12]. In a Stern-Gerlach arrangement with two spatially separated partial waves of the microobject, covered by their respective detectors labelled 1 and 2, unitary evolution would result in the entangled quantum state

$$
|\Psi\rangle \Rightarrow c_{+}|+\rangle \psi_{+}^{(1)} \psi_{-}^{(2)}+c_{-}|-\rangle \psi_{-}^{(1)} \psi_{+}^{(2)} ;
$$

measurement would take place by the quantum state collapsing into one of the above terms. Either of the choices implements full anticorrelation of the detectors.

The main difference with the present approach is that whereas in orthodox quantum mechanics $\psi_{+}(i)$ and $\psi_{-}(i)$ $(i=1$ or 2$)$ are macroscopically distinct, our approach considers them as being lumped together by gravity, which is, however, acting independently on the two detectors. Accordingly, the two non-collapsing Everettean branches of the superposition are macroscopically indistinguishable, moving as a single point of the configuration space spanned by some measurement coordinates $\xi_{1}$ and $\xi_{2}$ of the two detectors which, however, take their classical random choices independently. Apparently, there is nothing in the formalism to exclude that both detectors should choose to be active, or both quiescent. The same refers to the much-tested EPR case [11]: if detectors take independent random choices, there is nothing in the scheme to force anticorrelations.

However, detector anticorrelation is a strong effect. If, like randomness itself, it has a dynamical origin, that origin must be a strong force, destabilizing the "diagonal" in the two-detector configuration space [29] and driving the system towards one of the possible end points with one detector active, one quiescent (see FIG. (1). To make it more embarrassing, this is not a genuine configuration space, detector anticorrelations being completely detached from the detectors' location in three-dimensional space. This is fully analogous to the situation in the stochastic reduction scheme 3 : if there is a dynamical process behind the correlations, whether it is described by an external random force [30] or by a nonrandom one as suggested here, it must be related to the whole entangled state vector; so far, with no better representation in sight than Hilbert-space itself. Where to look in the theory for such a force, remains open to speculation for the moment. My guess and hope, already hinted at in the introduction to this Letter, is a possible modification of the Newton-Schrödinger scheme [] in such a way that the source of gravity should include quantum entanglement. The possibly related open issues of dark matter and Einstein's cosmological term may add to the identikit of the desired modification.

\section{CONCLUSIONS}

In this paper a thorough critical study of the NewtonSchrödinger approach to the quantum measurement problem has been presented, under the ambitious scope of supporting a no-split no-collapse scenario, with emergent randomness. The qualitative picture formed has extremely promising features. The scheme offers a transparent characterization of classicality, which is selfsustaining; it eliminates the paradoxical features of the Everett approach since macro-objects do not suffer macro-separation; finally, it offers a dynamical route to emergent quantum randomness.

However, there are serious doubts preventing immediate application of the scheme. First of all, gravity, as we know it from macroscopic measurements, is too weak for the purpose. Therefore its eventual short-range enhancement, theoretically proposed but not yet confirmed by straightforward experiments, may be crucial for classicality. In turn, if the present scheme gets further support, that can serve as an indirect indication for short-range gravity enhancement.

More far-reaching is the issue of the unexplained origin of entanglement-based detector anticorrelations, which calls for a refined characterization of the source of gravity, with nonlocal forces related to quantum entanglement in a way to be found.

In view of the discussion in the previous section, that is a formidable task. In the same time, until explicitly falsified, it is a logically open - even if very narrow way out of the dual description of the quantum-classical world. 


\section{ACKNOWLEDGMENTS}

I thank Lajos Diósi for giving me innumerable explanations about various approaches, as well as Abner Shimony and Nicolas Gisin for important criticism on various preliminary versions. I am indebted to Anton Zeilinger for supplying reference [18]. Discussions with my MSc student István Varga-Haszonits helped clarifying some points. This work has been partially supported by the Hungarian Research Foundation (grants OTKA T 029544 and T 034832).

\section{APPENDIX A}

The values of the parameters appearing in Eq. (3) are given in the for a spherical body of radius $R$. Here, motivated by the case of an electron liquid in a metallic lead connecting a detector to a voltmeter, we give them for the case of a rectangular slab of cross-section $a \times b$ (diameter $d$ ) and length $L \gg a, b$ :

$$
V_{g r}^{0}=-2 \ln (L / d) G M^{2} / L
$$

and

$$
\omega_{g r}^{2}=G \varrho_{0} \frac{d}{L} f\left(\frac{a}{b}\right)
$$

where

$$
\begin{array}{r}
f(x)=\frac{2}{x+1 / x}\left(2 \sqrt{1+x^{-2}} \ln \left(x+\sqrt{1+x^{2}}\right)\right. \\
+2 \sqrt{1+x^{2}} \ln \left(x^{-1}+\sqrt{1+x^{-2}}\right) \\
\left.-\frac{2}{3}\left(2+x^{2}+x^{-2}-x \sqrt{1+x^{2}}-x^{-1} \sqrt{1+x^{-2}}\right)\right)
\end{array}
$$

In particular, $f(x)=4.205$ for $x=1$ and $(4 / x) \ln x$ for $x \gg 1$.
[1] The acknowledged classics on related issues is Quantum Theory and Measurement, J. A. Wheeler and W. H. Zurek, eds. (Princeton University Press, Princeton 1983).

[2] J. von Neumann, Mathematische Grundlagen der Quantenmechanik, (Springer, Berlin, 1932); English translation: Mathematical Foundations of Quantum Mechanics, (Princeton University Press, 1955).

[3] For a most recent review, see A. Bassi and G. C. Ghirardi, Phys. Rep. 379, 257 (2003).

[4] Connecting gravitation with wave-vector collapse has a long history. Among the significant contributions, we mention R. P. Feynman, Lectures on Gravitation, (California Institute of Technology, Berkeley, 1962-1963); F. Károlyházi, Nuovo Cimento A 42, 390 (1966); F. Károlyházi, A. Frenkel and B. Lukács, in: Physics as Natural Philosophy, A. Shimony and H. Feshbach, eds. (MIT Press, Cambridge, 1982), p. 2; F. Károlyházi, A. Frenkel and B. Lukács, in: Quantum Concepts in Space and Time, R. Penrose and C. J. Isham, eds. (Oxford University Press, Oxford, 1986) p. 109; L. Diósi, Phys. Lett. A 120, 377 (1987); L. Diósi, Phys. Rev. A 40, 1165 (1989); G. C. Ghirardi, R. Grassi and A. Rimini, Phys. Rev. A 42, 1057 (1990); R. Penrose, General Relativity and Gravitation 28, 581 (1996); J. Anandan, Found.Phys. 29, 333-348 (1999).

[5] I. Bialynicki-Birula and J. Mycielski, Ann. Phys. (N.Y.) 100, 62 (1976); S. Weinberg, Phys. Rev. Lett. 62, 485 (1989), Ann. Phys. (N.Y.) 194, 336 (1989).

[6] A. Shimony, Phys. Rev. A 20, 394 (1979); the idea works for nonlinearities of unspecified origin; experimental tests have been carried out for neutrons by C. G. Shull, D. K. Atwood, J. Arthur and M. A. Horne, Phys. Rev. Lett. 44, 765 (1980), and by R. Gähler, A. G. Klein and A. Zeilinger, Phys. Rev. A 23, 1611 (1981).

[7] First described by L. Diósi, Phys. Lett. A 105, 199 (1984), the scheme was recently rediscovered and elaborated by R. Penrose, Phil. Trans. R. Soc. Lond. A 356, 1927 (1998); I. Moroz, R. Penrose and K. P. Tod, J. Class.
Quant. Grav. 15, 2733 (1998); I. Moroz and K. P. Tod, Nonlinearity 12, 201 (1999).

[8] For a readable account on cosmological generation of classical states of matter, see e. g. A. D. Linde, Inflation and Quantum Cosmology, (Academic Press, Boston, 1990).

[9] H. Everett III, Rev. Mod. Phys. 29, 454 (1957).

[10] M. Born, Z. f. Physik 37, 863 (1926).

[11] A. Einstein, B. Podolsky and N. Rosen, Phys. Rev. 47 (1935) 777; for a recent review of the experimental situation, see A. Zeilinger, Rev. Mod. Phys. 71 (1999) S288.

[12] A. Einstein, Fifth Solvay Conference, 192\%, discussed in: M. Jammer, The Philosophy of Quantum Mechanics, (Wiley-Interscience Publications, New York, 1974), pp. 115-117. A careful experimental test of that feature has been reported in Ref. 18 .

[13] Y. Aharonov and D. Bohm, Phys. Rev. 115, 485 (1959); B. E. Allman, A. Cimmino, A. G. Klein, G. I. Opat, H. Kaiser and S. A. Werner, Phys. Rev. Lett. 68, 2409 (1992).

[14] For destructive photon detection, where the final state of the microobject is the common vacuum, the same effect is ensured by environment-induced decoherence, on which the most recent review is W. H. Zurek, Rev. Mod. Phys. 75, 715 (2003). For extensive reviews, covering other competing approaches as well, see D. Giulini, E. Joos, C. Kiefer, J. Kupsch, I.-O. Stamatescu, H. D. Zeh, Decoherence and the Appearance of a Classical World in Quantum Theory, (Springer, Berlin, 1996); a recent careful analysis of related experiments can be found in A. J. Leggett, J. Phys.: Condens. Matter 14 (2002) R415.

[15] The idea to characterize detectors through their metastability appears again and again over the years. A particularly clear presentation is in N. G. van Kampen, Physica A 153, 97 (1988).

[16] S. M. Sze, Physics of Semiconductor Devices (2nd ed., Wiley-Interscience Publications, New York, 1981), Sec. 13.4 .

[17] N. F. Mott, Proc. Roy. Soc. A126, 79 (1929). 
[18] P. Grangier, G. Roger and A. Aspect, Europhys. Lett. 1, 173 (1986).

[19] N. Arkani-Hamed, S. Dimopoulos, and G. Dvali, Phys. Lett. B 429, 263 (1998); N. Arkani-Hamed, S. Dimopoulos, G. Dvali, and N. Kaloper, Phys. Rev. Lett. 84, 586 (2000).

[20] S. Dimopoulos and G. F. Giudice, Phys. Lett. B 379, 105 (1996); D. B. Kaplan and M. B. Wise, J. High Energy Phys. 08, 037 (2000).

[21] A not exhaustive list is: C. D. Hoyle et al., Phys. Rev. Lett. 86, 1418 (2001); J. Chiaverini et al., Phys. Rev. Lett. 90, 151101 (2003); J. C. Long et al., Nature 421, 922 (2003); most recent reviews are: I. Antoniadis, in Aspects of Quantum Gravity, ed. C. Lämmerzahl, Lecture Notes in Physics, Springer, Berlin 2003; J. C. Long and J. C. Price, CR Physique 4, 337 (2003).

[22] So far untested proposals are: E. Fischbach, D. E. Krause, R. S. Decca, and D. López, Phys. Lett. A 318, 165 (2003); A. Frank, P. Van Isacker, and J. GómezCamacho, nucl-th/0305029

[23] M. Arndt, O. Nairz, J. Vos-Andreae, C. Keller, G. van der Zouw and A. Zeilinger, Nature 401, 680 (1999); B. Brezger, L. Hackermüller, S. Uttenthaler, J. Petschinka, M. Arndt and A. Zeilinger, Phys. Rev. Lett. 88, 100404 (2002); L. Hackermüller, S. Uttenthaler, K. Hornberger,
E. Reiger, B. Brezger, A. Zeilinger, and M. Arndt, Phys. Rev. Lett. 91, 090408 (2003).

[24] I. Varga-Haszonits and T. Geszti, unpublished.

[25] N. Gisin, Phys. Lett. A 143, 1 (1990); J. Polchinski, Phys. Rev. Lett. 66, 397 (1991).

[26] For related discussions see M. Czachor and H.-D. Doebner, Phys. Lett. A 301, 139 (2002); A. Kent, quant-ph/0204106

[27] A. Shimony, in: Foundations of Quantum Mechanics in the Light of New Technology, ed S. Kamefuchi (Physical Society of Japan, Tokyo, 1983).

[28] This argument is not related to the sophisticated construction termed "one-particle nonlocality": see S. M. Tan, D. F. Walls and M. J. Collett, Phys. Rev. Lett. 66, 252 (1991); L. Hardy, Phys. Rev. Lett. 73, 2279 (1994); with comments by L. Vaidman, ibid. 75, 2063 (1995), D. M. Greenberger, M. A. Horne and A. Zeilinger, ibid. 75, 2064 (1995), and reply by L. Hardy: ibid. 75, 2065 (1995).

[29] A deflecting force may appear in the disguise of destructive interference (or vice versa), see e.g. C. A. Mead, Rev. Mod. Phys. 64 (1992) 51; Y. Aharonov, T. Kaufherr, S. Popescu and B. Reznik, Phys. Rev. Lett. 80 (1998) 2023.

[30] N. Gisin, Phys. Rev. Lett 52 (1984) 1657. 